\title{
The Impact of New EUV Diagnostics on CME-Related Kinematics
}

\author{
Scott W. McIntosh • Bart De Pontieu • Robert J. Leamon
}

Received: 30 November 2009 / Accepted: 26 February 2010 / Published online: 23 March 2010

(C) Springer Science+Business Media B.V. 2010

\begin{abstract}
We present the application of novel diagnostics to the spectroscopic observation of a Coronal Mass Ejection (CME) on disk by the Extreme Ultraviolet Imaging Spectrometer (EIS) on the Hinode spacecraft. We apply a recently developed line profile asymmetry analysis to the spectroscopic observation of NOAA AR 10930 on 14-15 December 2006 to three raster observations before and during the eruption of a $1000 \mathrm{~km} \mathrm{~s}^{-1}$ halo CME. We see the impact that the observer's line-of-sight and magnetic field geometry have on the diagnostics used. Further, and more importantly, we identify the on-disk signature of a high-speed outflow behind the CME in the dimming region arising as a result of the eruption. Supported by recent coronal observations of the STEREO spacecraft, we speculate about the momentum flux resulting from this outflow as a secondary momentum source to the CME. The results presented highlight the importance of spectroscopic measurements in relation to CME kinematics, and the need for full-disk synoptic spectroscopic observations of the coronal and chromospheric plasmas to capture the signature of such explosive energy release as a way of providing better constraints of CME propagation times to L1, or any other point of interest in the heliosphere.
\end{abstract}

Remote Sensing of the Inner Heliosphere

Guest Editors: M.M. Bisi, and A.R. Breen.

Electronic supplementary material The online version of this article

(doi:10.1007/s11207-010-9538-z) contains supplementary material, which is available to authorized users.

S.W. McIntosh $(\bowtie)$

High Altitude Observatory, National Center for Atmospheric Research, P.O. Box 3000, Boulder, CO 80307, USA

e-mail: mscott@ucar.edu

B. De Pontieu

Lockheed Martin Solar and Astrophysics Lab, 3251 Hanover St., Org. ADBS, Bldg. 252, Palo Alto, CA 94304, USA

e-mail: bdp@Imsal.com

R.J. Leamon

Department of Physics, Montana State University, Bozeman, MT 59717, USA

e-mail: robert.j.leamon@nasa.gov 
Keywords Spectral line - Intensity and diagnostics · Chromosphere · Active · Active regions · Magnetic fields · Coronal mass ejections · Low coronal signatures · Solar wind · Disturbances

\section{Introduction}

In this short forward-looking and speculative paper, we present an extended analysis of observations of NOAA AR 10930 from the Extreme-Ultraviolet Imaging Spectrometer (EIS; Culhane et al., 2007) on Hinode (Kosugi et al., 2007) between 19:00 UT 14 December 2006 and 06:00 UT 15 December 2006. This time period saw an X-Class flare and a fast (planeof-the-sky speed ${ }^{1} \sim 1000 \mathrm{~km} \mathrm{~s}^{-1}$ ) halo CME and coronal dimming event (e.g., Kahler and Hudson, 2001; Attrill et al., 2008) that emanated from this complex active region at around 20:12 UT.

We expand on the analysis of McIntosh et al. (2007), McIntosh (2009), and McIntosh, Burkepile, and Leamon (2009), exploiting the rare detailed spectroscopic measurements of dimming region evolution that were first studied by Harra et al. (2007). EIS provides a tantalizing look at the dynamic behavior of EUV emission lines over the course of the eruption. The interpretation of the dynamic evolution of the non-thermal line widths presented forms an extension of the challenge posed by McIntosh (2009) to the rapidly increasing sophistication of numerical CME models: specifically that they need to cope with the complex thermodynamics of the CME source region. Clearly, the relationship between the dimming region and CME is one that grows considerably when only narrowband spectroscopic observations are considered. Therefore, rigorously establishing the poorly understood physical connection between CMEs and coronal dimmings using detailed spectroscopic measurement is a must. Fortunately, there is an ever-growing list of investigations in the literature (Harrison and Lyons, 2000; Imada et al., 2007; Harra et al., 2007; Bewsher, Harrison, and Brown, 2008; Jin et al., 2009; Harra, 2009) that, we hope, will continue to expand with ongoing EIS observation and the launch of IRIS, the Interface Region Imaging Spectrograph, ${ }^{2}$ in late 2012.

In the following sections we describe the observations used and the technique developed to assess the asymmetry of the emission line profiles (e.g., De Pontieu et al., 2009; McIntosh and De Pontieu, 2009), new imaging results that validate the spectral analysis, how we interpret those datasets, and finally, speculate on the implications of the results on the kinematic properties of the CME itself.

\section{Observations and Analysis}

The dataset of interest comprises three spectroheliogram "raster" observations (19:2021:34 UT, 01:15-03:30, 04:10-06:24 UT), targeted at the trailing edge of the active region that is the source of the event studied. The EIS observations are reduced using the IDL Solarsoft (Freeland and Handy, 1998) eis_prep algorithm which corrects for cosmic ray

\footnotetext{
${ }^{1}$ The CME properties were automatically derived from SOHO/LASCO data by the NASA/GSFC CDAW (http://cdaw.gsfc.nasa.gov/) and the Royal Observatory of Belgium/SIDC CACTUS (http://www.sidc.be/ cactus/; Robbrecht and Berghmans, 2004) catalogues.

${ }^{2}$ See the IRIS website (http://iris.Imsal.com/) for more information about the mission.
} 

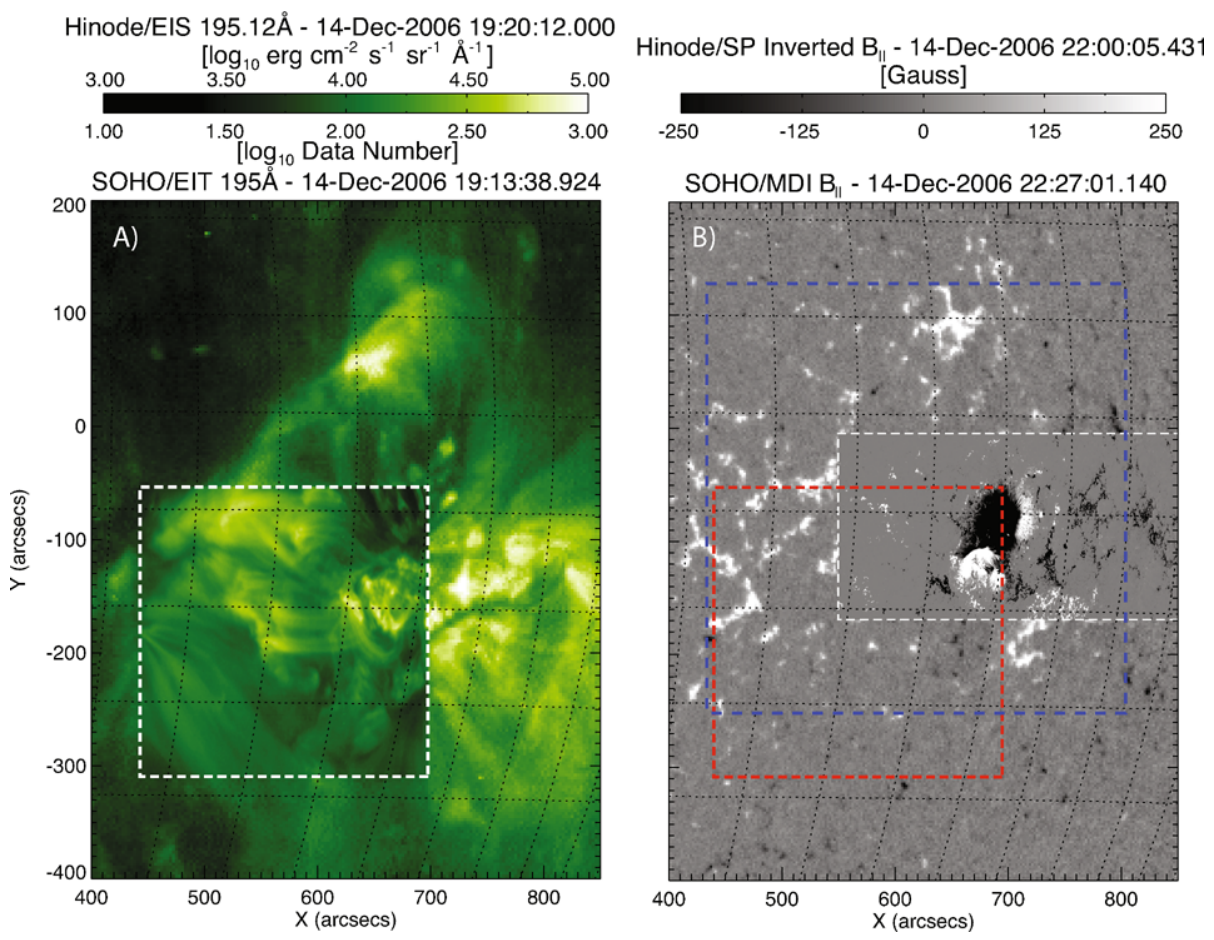

Figure 1 Representative pre-CME images of NOAA AR 10930 from SOHO and Hinode. Panels A and B show the full-field EIT $195 \AA$ and MDI LOS magnetogram with Hinode/EIS and Hinode SOT/SP images inlaid for reference respectively. The red and blue dashed regions in the right panel respectively show the EIS field of view and region of the composite MDI/SP magnetogram extrapolation used to provide context below.

hits, hot pixels, detector bias, and dark current, and converts data numbers to intensities (in $\operatorname{erg~cm}{ }^{-2} \mathrm{~s}^{-1} \mathrm{sr}^{-1} \AA^{-1}$ ).

Each EIS raster is comprised of 256 horizontal (West to East) mirror mechanism steps with the $1^{\prime \prime}$ slit at a spacing of $1^{\prime \prime}$ and a height of $256^{\prime \prime}$ and has information in nine 24-pixel wide spectral windows. At a spectral resolution of $22.3 \mathrm{~m} \AA$, and wavelength of $195 \AA$, one pixel on the detector is equivalent to a velocity of $\sim 34 \mathrm{~km} \mathrm{~s}^{-1}$. Panel A of Figure 1 shows the region surrounding AR 10930 provided by the $195 \AA$ passband image of SOHO/EIT (Delaboudinière et al., 1995) taken at 19:13 UT which is inlaid with the peak line intensity of the EIS $195 \AA$ raster.

The SOT Spectro-Polarimeter (SP) rastered the region of interest twice during the time of interest (14 December 2006 22:00 - 23:03 UT and 15 December 2006 05:45 - 06:48) taking 1000 stepped measurements per raster in Stokes I, Q, U, and V in Fe I 6301.5, 6302.5 and binning two-by-two pixels on board to give an effective (linear) spatial scale of $0.32^{\prime \prime}$. The SP data were reduced using the standard settings of the sp_prep routine in the SOT software tree. The Stokes polarization signals measured by SP are inverted into a set of physical parameters that describe the vector magnetic field (field strength $B$, inclination from the local normal $\psi$, azimuth $\phi$, filling factor $S_{\alpha}$, etc.) and local plasma conditions using the approach of Skumanich and Lites (1987): seeking to simultaneously minimize the least-squares fit of four Stokes profiles with analytic descriptions of the polarization signals under the Zeeman effect in a Milne-Eddington atmosphere. The Stokes inversion shown 
here is executed using the MERLIN algorithm (Lites et al., 2007) that has recently been developed at NCAR under the framework of the Community Spectro-polarimetric Analysis Center (CSAC; http://www.hao.ucar.edu/projects/csac/) for use with SP. The full SP Stokes vector maps are shown, for reference, in McIntosh, Burkepile, and Leamon (2009).

An example of an SP line-of-sight (LOS) magnetogram, constructed from the inverted Stokes I, Q, U, and V measurements, allows us to look in detail at the LOS field: $B_{\|}=$ $S_{\alpha} B \cos \psi$ and is inlaid in the full-disk LOS magnetogram from SOHO/MDI (Scherrer et al., 1995) and shown in panel B of Figure 1. Note that we have used the full-disk SOHO images/magnetograms to align the sub-field Hinode observations of EIS and SOT - this allows us to correct the pointing of the Hinode data to within $3^{\prime \prime}$. Indeed, using the SOHO data as a pointing reference has the added advantage of allowing good coalignment between EIS and SOT. In Figure 1B, the sub-field covered by EIS is indicated by a red dashed outline while the blue dashed outline marks a region for which we extract a potential field extrapolation for the purpose of later discussion (see Figure 5).

\subsection{R-B Analysis}

Following the description of De Pontieu et al. (2009) and McIntosh and De Pontieu (2009) we perform a 'Red Minus Blue' (R-B) profile asymmetry analysis on spectral lines in the EIS data that are not significantly impacted by spectral blends in the relatively narrow (24 pixel) spectral windows used, i.e., the Fe XIII $202 \AA$ and Fe XIV $274 \AA$ lines. The R-B analysis involves several steps. First we fit a single Gaussian shape to the emission line profile at each pixel to establish the line centroid. Once determined, we sum the amount of emission in narrow ( $\sim 24 \mathrm{~km} \mathrm{~s}^{-1}$ wide) spectral regions symmetrically placed about the determined centroid in a line profile interpolated to ten times the spectral resolution. We then subtract the red and blue wing contributions to the interpolated profile to make a filtergram sampling a particular velocity range. A positive value of R-B indicates an asymmetry in the red wing of the line, which we can interpret as the signature of excess down-flowing material at that velocity while, conversely, a negative value of R-B would indicate an excess of up-flowing material.

Figures 2 and 3 show the peak line intensities (top row), R-B at $110 \mathrm{~km} \mathrm{~s}^{-1}$ (middle row), and at $160 \mathrm{~km} \mathrm{~s}^{-1}$ (bottom row) for the Fe XIII $202 \AA$ and Fe XIV $274 \AA$ lines respectively for the three phases of the dimming observed (left column - 19:20-21:34 UT; middle 01:15-03:30 UT; right - 04:10-06:24 UT). We note that movies of the complete velocity range are available online and the presence of the vertical anomaly in the pre-eruption spectroheliograms that were caused by a couple of bad detector read outs at a few slit positions.

The three movies supporting Figure 3 (Movies 1 through 3) show the progression of the R-B analysis for the Fe XIV $274 \AA$ line from $40 \mathrm{~km} \mathrm{~s}^{-1}$ through $200 \mathrm{~km} \mathrm{~s}^{-1}$ in context with the peak line intensity, $1 / \mathrm{e}$ line width, and (relatively calibrated) Doppler velocity over the course of the dimming event observed by EIS. In all of the movies we notice the general correspondence between the regions of enhanced line broadening, significant blue wing asymmetries and the darkest coronal loop structures as was noted, and discussed, in McIntosh and De Pontieu (2009). During the dimming event we notice the extension of the excess non-thermal broadening of the line into the regions far from the magnetic footpoints (and associated blue wing asymmetry) of the dimming region. It is this extended, and dynamic, variation of the line widths that McIntosh (2009) attributed to the growth of Alfvén waves on the now open, less dense, coronal magnetic field lines behind the CME. However, De Pontieu et al. (2009) and McIntosh and De Pontieu (2009) suggested that the blueward asymmetries in a wide variety of lines formed at transition region and coronal temperatures 


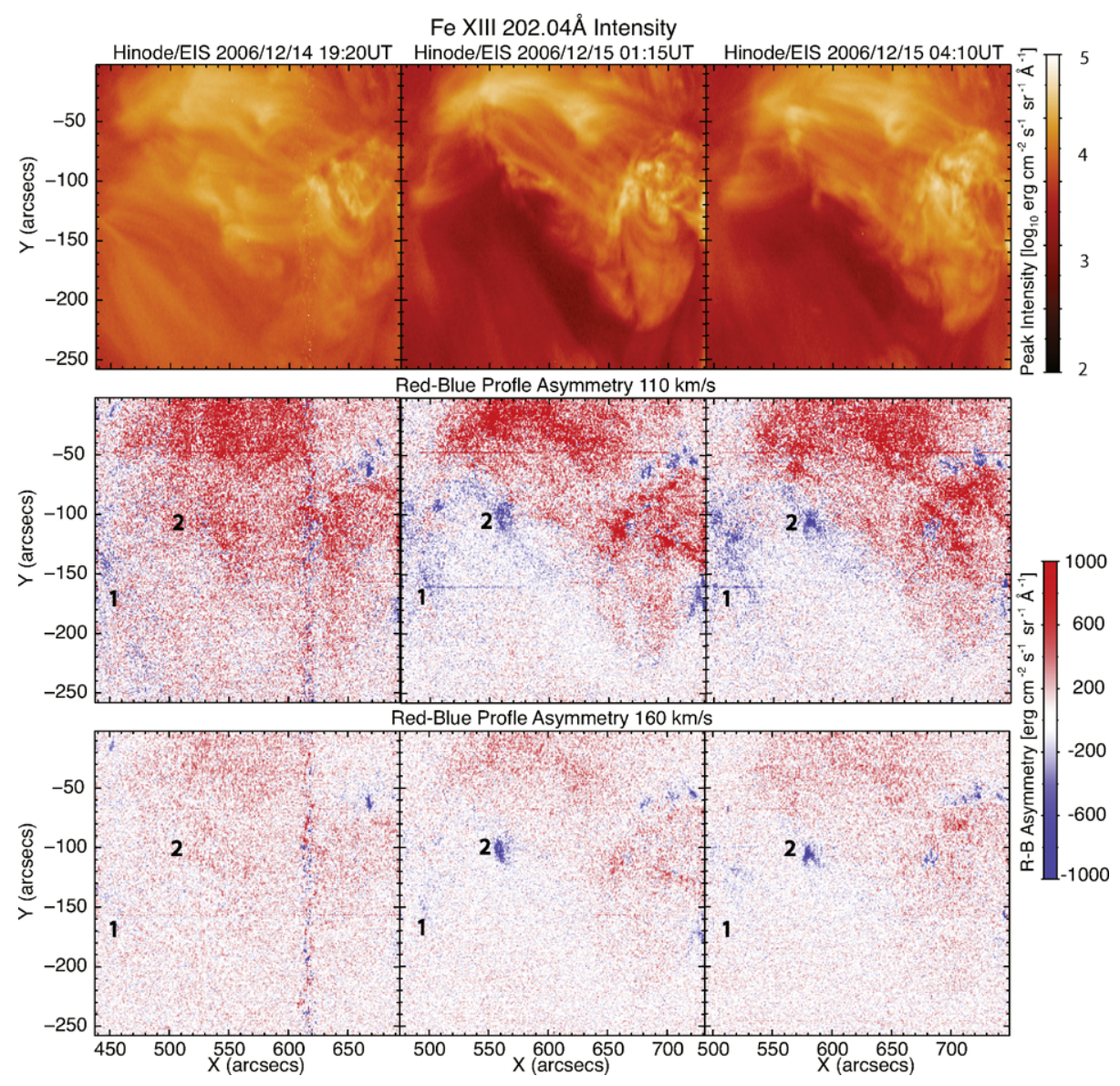

Figure 2 Three phases of evolution of the CME as observed in the Fe XIII 202 A emission line peak intensity (top row) and R-B analysis maps (see text for details of the R-B method) showing the R-B maps at $110 \mathrm{~km} \mathrm{~s}^{-1}$ (middle row) and $160 \mathrm{~km} \mathrm{~s}^{-1}$ (bottom row). The color of the R-B maps indicate the net direction of the plasma motion at that velocity, red regions suggest net downflow, while blue regions suggest net upflow. The numbers $\mathbf{1}$ and $\mathbf{2}$ in the middle and lowest rows indicate loop complexes at location 1 and location 2, from where the bulk of the dimming originates.

may be caused by upflows from chromospheric spicules that are associated with hot upflows at $\sim 100 \mathrm{~km} \mathrm{~s}^{-1}$. Given this interpretation and our observation of excess blueward asymmetries where the line widths are enhanced, we would expect that a significant portion of the excess broadening at the magnetic footprint of these regions is caused by the presence of high-velocity spicular outflows. Correctly apportioning the observed broadening there to spicular outflows or larger amplitude Alfvénic motions of the roots of the magnetic field lines is difficult without higher temporal, spatial, and spectral resolution data. This is an issue we will return to below.

\section{Results}

The temporal evolution of the line intensity is shown in the top rows in Figures 2 and 3, where in the central panels we see a sudden dimming to the South-East of the active region 


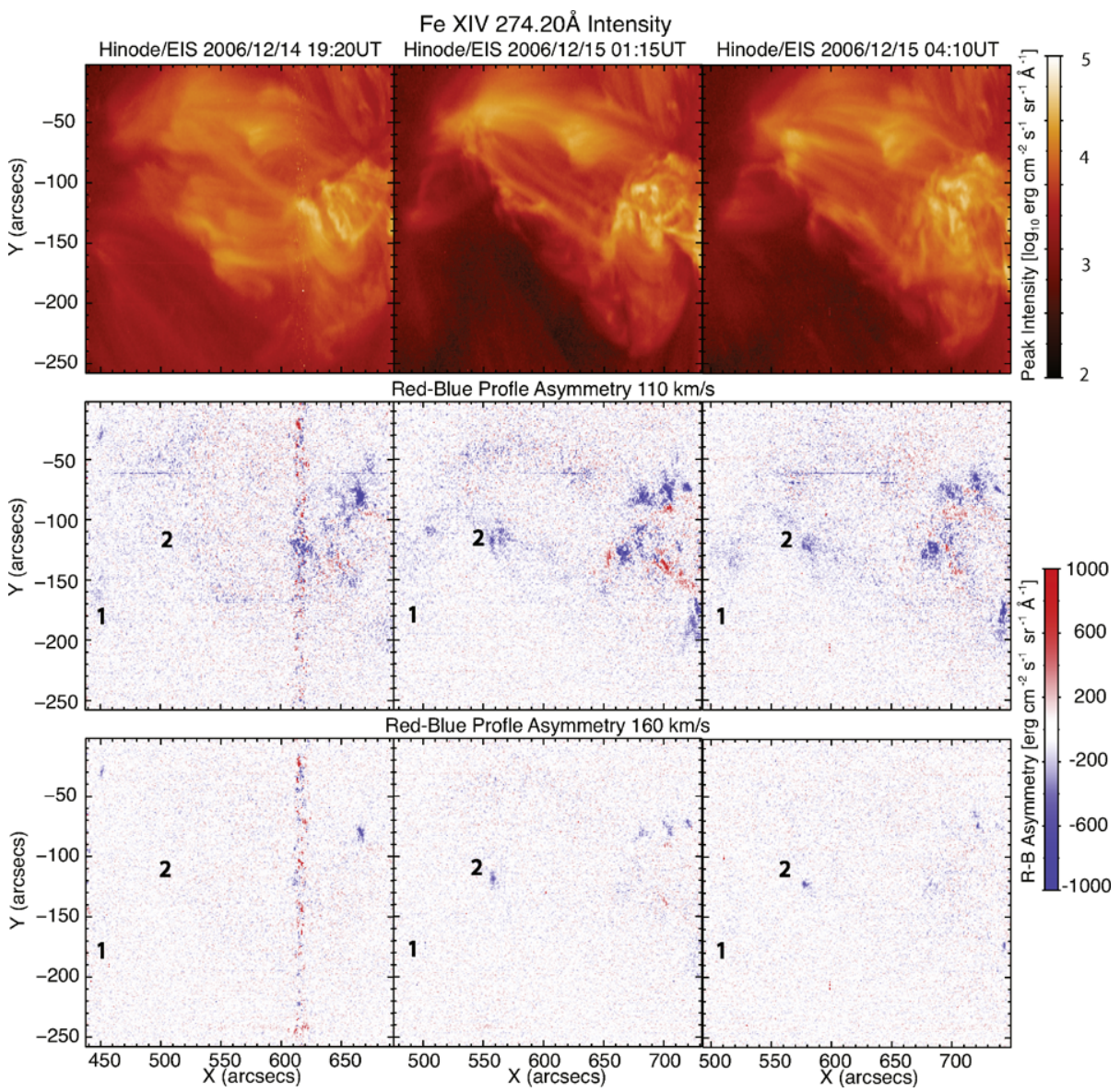

Figure 3 Three phases of evolution of the CME as observed in the Fe XIV 274 A emission line peak intensity (top row) and R-B analysis maps (see text for details of the R-B method) showing the R-B maps at $110 \mathrm{~km} \mathrm{~s}^{-1}$ (middle row) and $160 \mathrm{~km} \mathrm{~s}^{-1}$ (bottom row). The color of the R-B maps indicate the net direction of the plasma motion at that velocity, red regions suggest net downflow, while blue regions suggest net upflow. The numbers 1 and $\mathbf{2}$ in the middle and lowest rows indicate loop complexes at location 1 and location 2, from where the bulk of the dimming originates. Movies 1, 2, and 3 of the online material are provided to support the three columns of the figure from left to right respectively.

between the first and second rasters; there is a $\sim 75 \%$ reduction in intensity in that region between the first two images. By the third raster, we see some filling of the region has taken place close to the active region (McIntosh et al., 2007). We see that the bulk of the dimming originates in the loop complexes that originate in the positive polarity flux domains at $[x, y]=\left[450^{\prime \prime},-170^{\prime \prime}\right]$ (location 1) and $\left[530^{\prime \prime},-130^{\prime \prime}\right]$ (location 2) in Figures 2 and 3. These loop systems span the South-Eastern portion of the active region connecting it to the negative polarity flux at $\left[650^{\prime \prime},-140^{\prime \prime}\right]$, the sunspot, and surrounding flux. Aspects of this connectivity are shown in Figure 5.

Prior to the eruption (the first columns of Figures 2 and 3) we see a very weak blue wing asymmetry in Fe XIII at $110 \mathrm{~km} \mathrm{~s}^{-1}$ in location 1 that is fainter still in Fe XIV, but there is no obvious signal in the $160 \mathrm{~km} \mathrm{~s}^{-1}$ asymmetry map there. There are regions of 
high-velocity blue asymmetries (suggestive of upflows) present in the active region prior to the eruption, however, and perhaps the most prevalent is that at $\left[660^{\prime \prime},-130^{\prime \prime}\right]$, which is on the darker loops coming out of the North of the sunspot where we have seen similar multi-thermal outflow signatures before ( $c f$. McIntosh, 2009). We also note the clear signature of hot (Fe XIV) upflows in the negative polarity region at the southern portion of the active region $\left[630^{\prime \prime},-160^{\prime \prime}\right]$, while it is puzzling that no clear signature exists in the Fe XIII emission.

After the eruption (the central columns of Figures 2 and 3), when the corona behind the CME is open, we see that the asymmetry maps have changed considerably, signatures of strong upflows are now seen in the flux concentrations at the bottom of the dimming region and over more of the active region. The most conspicuous of the new upflow regions in both spectral lines at 110 and $160 \mathrm{~km} \mathrm{~s}^{-1}$ is rooted in location 2, now at $\left[560^{\prime \prime},-150^{\prime \prime}\right]$ (in the central column, $\left[580^{\prime \prime},-150^{\prime \prime}\right]$ in the right column), the small flux concentration $50^{\prime \prime}$ to its East and the positive polarity region at the other end of the dimming region $\left[720^{\prime \prime},-220^{\prime \prime}\right]$ (in the central column, $\left[740^{\prime \prime},-220^{\prime \prime}\right]$ in the right column).

In both lines, but certainly clearer in $274 \AA{ }^{3},{ }^{3}$ the southern portion of the sunspot region (e.g., $\left[670^{\prime \prime},-170^{\prime \prime}\right]$ ) is showing larger contiguous regions of upflow. Interestingly, this location was studied in detail by McIntosh, Burkepile, and Leamon (2009) and it was noted that the penumbral structure of the region disappeared after the eruption, not to return while the region was visible on the disk. This observation will enter into our deliberations of what we are seeing and why (below). We also note that, in Fe XIII, we still see the weak upflow signal from location 1 although it appears to become increasingly extended as the active region complex rotates towards the limb.

Of course, in addition to the upflows that become visible over the course of the event, we see high-speed hot downflows in certain locations. These are most clearly identified as the bright red regions (e.g., at [700", 190"]) most easily seen in Fe XIV (the central column of Figure 3). We see that these downflows occur at the bottoms of the bright, compact, posteruption loops that form as the corona starts to close behind the CME. Incidentally, these downflows are reduced in the later phase of the event.

This analysis, maps and associated movies, provide further interesting insight into the reaction of the coronal plasma to the morphology change imposed on it by the eruption of the CME. In the following section we will attempt to place this analysis in context and provide some insight into the possible impact of the on-disk changes to the CME itself.

\subsection{STEREO Images the Relentless Outflow}

As has been demonstrated by McIntosh and De Pontieu (2009) the significant line profile asymmetries observed here have been connected with the visual appearance of plasma "blobs" that are visible in broadband coronal imaging diagnostics from TRACE, Hinode/XRT and the STEREO spacecraft. In Figure 4 and the accompanying movie (Movie 4) we see a small, asymmetric coronal dimming event that was observed in the Fe IX/X $171 \AA$ passband by SECCHI/EUVI (Howard et al., 2008) on STEREO "Behind" on 12 February 2009 as part of a special quadrature observation sequence. During this sequence a $171 \AA$

\footnotetext{
${ }^{3}$ We suspect that, while the $202 \AA$ Fe XIII emission line is spectrally clean according to the EIS spectral atlas (Brown et al., 2008), there may well be a subtle, undocumented blend or background issue in the red wing of the line that is affecting the R-B analysis. Regardless of this issue, there is dominant blue wing signature in the magnetic regions post-eruption that is greater in magnitude than this "contamination". We are aware of the issue with this line and are exploring multiple EIS datasets to identify what is going on.
} 


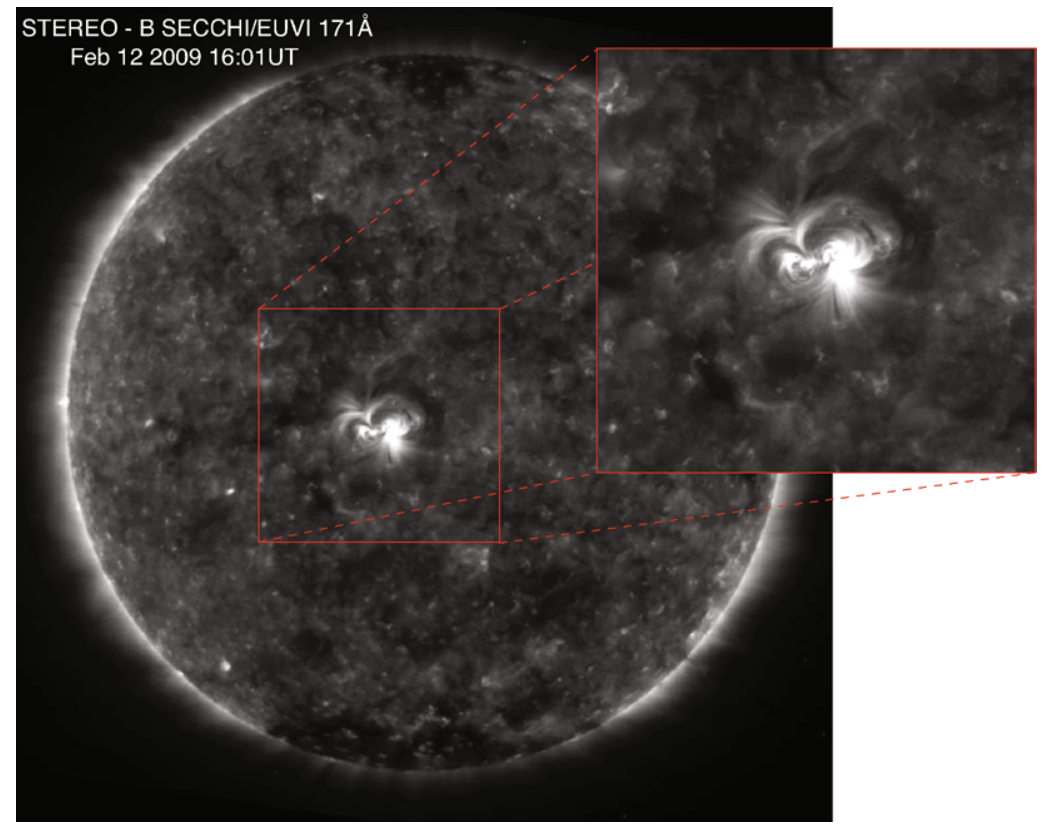

Figure 4 STEREO SECCHI/EUVI B image taken at 16:01 UT on 12 February 2009. The movie that accompanies this figure shows four hours of evolution in the small active region AR11012 in the figure inset at a cadence of $95 \mathrm{~s}$. The signature of the plasma "blobs" is clearly visible in the movie as a bursty outflow that appears to travel along the magnetic loop structures that comprise the AR. These blobs are visibly traveling outward from the surface before and throughout the eruption.

image was taken every $95 \mathrm{~s}$. The purpose of including this intriguing observation in the present paper is two-fold. It highlights that the energy release into the corona, and solar wind through the magnetic regions of the lower atmosphere is relentless, and that EUV imagers can provide some valuable information about more than the morphology of the coronal plasma.

The small active region near the center of the disk (NOAA AR 11012) was a prolific source of plasma blobs, which, as McIntosh and De Pontieu (2009) has demonstrated, are rooted in the same location as the multi-thermal coronal emission line asymmetries, and dynamic Type-II spicules that display similar velocity characteristics. It is fortunate that while spectroscopic instruments like EIS are burdened by the line-of-sight sensitivity of the weak plasma emission due to the spicule driven mass outflow, broadband imagers do not suffer the same effects, as spicule driven events are unlikely to be Doppler shifted out of the passband. These discrete, quasi-periodic mass loading events are visible on most coronal loops provided that the net brightness of those loops is low enough (they can be long, or low density): then the 5\% quasi-periodic mass injections are quite easily visible on the loop system.

Prior to the eruption of the AR (at 18:07 UT) we readily see the relentless release of hot mass heading upward into the corona above. As the corona is opened by the small filament eruption, the loop system to the East of the small active region is forced outward. The locations tethering those stretched out field lines continue to show the transmission of blobs throughout the dimming event.

We expect that it may be possible to use this form of EUV imaging data to study in some detail the energetics and (quasi-)periodicity of the mass loading events occurring before, 
during and after an event. Our suspicion is that the quasi-periodicity of the mass loading events on those magnetic field lines and the apparent association of these events with Alfvén waves present an environment in which Alfvén wave dissipation can drive the resulting fast wind stream behind the CME. A similar assertion is made when considering blob-like activity in polar plumes and their impact on the fast wind in polar coronal holes (McIntosh et al., 2010). This is discussed in more detail below.

\section{Discussion}

It is clear from the discussion above that the R-B maps of the coronal plasma change dramatically over the course of the event studied. We should stress that places where upflows are seen after the eruption are also likely to be sources of upflow before the event (an assertion validated by the STEREO observations, albeit of a different event) and this factor is critical to understanding what we observe. The reason for this apparent "switch-on" in the spectroscopic measurements must be largely geometric, the viewing angle between the magnetic field direction (along which the flows occur) and the line-of-sight is critical for determining the appearance of these weak blue Doppler asymmetries in relation to the bulk of the line emission. On highly inclined field lines to the observer's line-of-sight we will see little of the field directed motion, $\cos \theta$ is small and the true velocity of the upflow component is shifted towards lower velocities by that factor, with the end result that the upflow emission becomes part of the bulk of the line, rendering it practically invisible to our analysis. ${ }^{4}$ Conversely, when the observer is looking straight down on one of these field lines, we are able to see both the peak (weak) emission and the blue wing contribution. Depending on the relative magnitude of the blue wing contribution, the net effect of fitting the line profile with a single Gaussian profile is a larger 1/e width (as discussed in McIntosh et al., 2010) and an additional weighting of the line centroid to the blue by a few $\mathrm{km} \mathrm{s}^{-1}$. This additional profile broadening in the magnetic footpoints of the dimming region (in terms of a single Gaussian fit) augments the line broadening reported by McIntosh (2009). We speculate that we may have a two-stage process of mass loading at the bottom of those field lines and release of Alfvén waves driven by the generation process of the mass loading events and/or the change in tension along the field line due to the mass loading. These waves then propagate outward.

It is important to note that the emission observed by EIS over these strong magnetic field regions has at least two components. The R-B analysis we present is just one way of validating the weaker outflow component, and one that does not prescribe a particular functional form to the distribution observed. While the excess wing emission looks, to all intents and purposes, Gaussian in nature we believe that multi-Gaussian fits to the data are informative and validate the presence of the high-velocity component, but they offer little in terms of the complete physical description of these potentially important velocity distributions.

A snapshot of the magnetic field geometry of this active region prior to the eruption is shown in Figure 5. Using the composite MDI/SP line-of-sight magnetogram shown in Figure 1 we compute the associated potential field and plot some of the field lines rooted in the blue dashed region to get a picture of magnetic connectivity in the region. Unfortunately, it is unlikely that this line-of-sight magnetogram evolution offers much insight into the subtleties of the field geometry change following the CME. While there is a clear impact on the

\footnotetext{
${ }^{4}$ A study of this active region complex crossing the solar disk, and its impact on R-B analysis, will be completed shortly and be presented in the literature.
} 


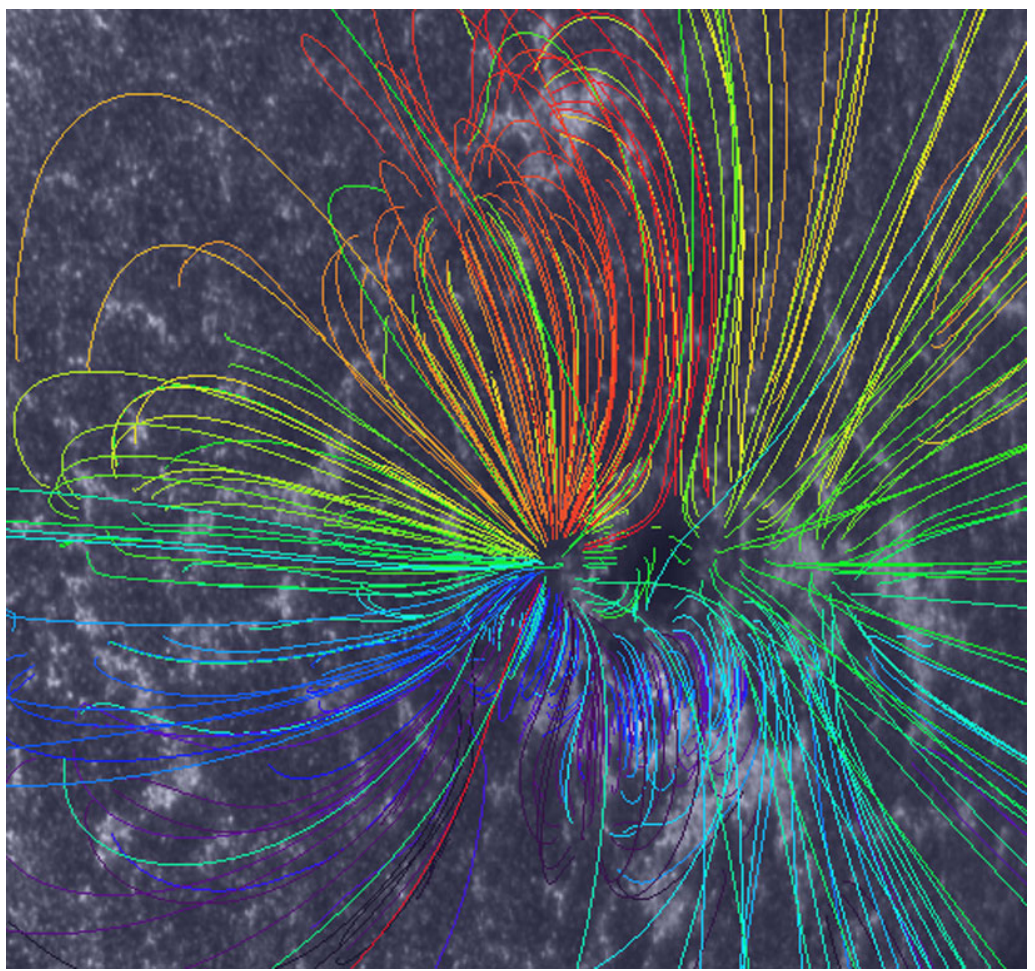

Figure 5 A snapshot of the potential field extrapolation of the active region studied before the eruption (AR 10930) using a combination of SOHO/MDI (14 December 2006 22:27 UT) and Hinode/SOT/SP inverted (14 December 2006 22:00-23:03 UT) magnetograms, see Figure 1. We have plotted the magnetic field lines that are rooted in the blue dashed region of Figure 1. The background image is that of the TRACE $1600 \AA$ passband and shows the mixture of dark spot structure and brighter plage emission distributed around this complex AR. We note that the considerable non-potentiality of this active region complex means that the field lines represented are only likely to be valid for the loops of the largest spatial extent.

chromospheric ( $\mathrm{Ca}$ II $\mathrm{H}$ ) and photospheric ( $\mathrm{G}$ band) emission in the vicinity of the sunspots (as reported in McIntosh, Burkepile, and Leamon, 2009), and in their penumbral structure following the eruption (as we have noted above), the spectro-polarimetric signal relative to that change is subtle and, to all practical purposes, hard to decipher (especially given the proximity of this region to the limb). The complexity of Stokes profile interpretation, and the resulting field representation, in the SP measurements is a topic we will leave to a more detailed subsequent investigation.

In light of the difficulties with interpreting the SP data we suggest to consider our R-B maps as an interpretative guide to the magnetic geometry. So, as the coronal magnetic field lines open up in response to the eruption of the CME we start to see a larger component of the upflow rooted ubiquitously in strong magnetic flux concentrations (De Pontieu et al., 2009; McIntosh and De Pontieu, 2009) along the line-of-sight observed with EIS. These outflows on the now open magnetic field lines are clearly oriented behind the CME, the plasma is hot $(>1 \mathrm{MK})$ and, as such has enough thermal pressure to overcome the Sun's gravitational field on those field lines (Parker, 1991) and is, to all intents and purposes, a stream of solar wind. The strength of the magnetic field at the bottom of these open regions is considerably larger than that of typical (equatorial) coronal holes (McIntosh, Davey, and Hassler, 2006) where 
ubiquitous outflows are readily observed in the magnetic network (De Pontieu et al., 2009; McIntosh, Leamon, and De Pontieu, in preparation) and may go some way to explaining why some coronal dimming events rooted in very strong magnetic flux regions have very fast CMEs that show little or no deceleration and have associated high wind streams behind them (e.g., Neugebauer, Goldstein, and Goldstein, 1997; Skoug et al., 2004).

Based on the analyses of De Pontieu et al. (2007, 2009) and McIntosh (2009) we suggest that the spicules that form the root of these upflow regions transport mass and Alfvénic wave energy behind the CME. It is expected that the significant Alfvénic energy present on the spicules, and coronal magnetic field lines, will propel the plasma outward from the Sun (e.g., Suzuki and Inutsuka, 2006; Cranmer, van Ballegooijen, and Edgar, 2007; Verdini and Velli, 2007). The presence of the mass and energy flux behind the CME poses an interesting challenge for our current understanding of CMEs, and particularly those that do not slow down from the $\sim 1000 \mathrm{~km} \mathrm{~s}^{-1}$ initial plane-of-the-sky observed speeds to the ambient wind speed of a few hundred kilometers per second through interplanetary space (e.g., Reinard and Biesecker, 2009): Does the quasi-periodically forced stream of mass and energy in the observed upflows have a significant impact on the momentum balance of the CME such that it would provide a continuous "push" for the material ahead to overcome the inertia of the plasma that the CME is propagating through?

In keeping with the tone of this article as one that is looking to future diagnostics of surface activity and their net impact on space weather, we estimate that the Atmospheric Imaging Array (AIA) of the Solar Dynamics Observatory (SDO) will have increased signalto-noise over SECCHI/EUVI of a factor of several. Coupled with the high image cadence $(10 \mathrm{~s})$, multi-thermal observations, and spatial pixels that are a factor of three smaller $\left(\sim 0.6^{\prime \prime}\right)$, we are entering a phase of solar physics when we will be able to investigate the root energetics of the outer solar atmosphere and the possible impact it has on associated ejecta. We anticipate that these observations can, and will, provide ideal boundary information for the increasingly sophisticated space weather modeling effort. In late 2012, IRIS will provide unprecedented spectra of these events in the transition region and upper chromosphere that will complement the observations of SDO/AIA and Hinode (if it is still operational) in such a way that we will be able to provide even stronger observational constraints towards resolving the puzzle of CME acceleration.

\section{Conclusion}

We have observed the "triggering" of high-velocity coronal outflows behind a CME that are rooted in the field of newly open strong magnetic flux regions. These upflows carry mass and Alfvénic wave energy outward from the Sun and, as such, are a potential momentum source for the CME while the coronal magnetic field is open.

We have observed a rapid switch of changes in "surface" thermodynamics from plasma heating to plasma forcing on the open magnetic topology and the relentless nature of the mass loading on those magnetic field lines at all phases of the eruption. Clearly, a larger sample of spectroscopically studied CMEs (preferably from high-cadence, full-disk spectral imaging instrumentation of the chromosphere and corona) are needed to study the true impact of this potential driver. While this paper is very speculative in nature, and we offer no solution to the issue at this point (sorry), the observations presented, and results discussed, give significant food for thought. In the near future observations such as those discussed herein will help us ascertain if the resulting fast solar wind outflow behind the CME impacts the kinematics of the disturbance as it travels into the inner heliosphere. 
Acknowledgements SWM is supported by NSF ATM-0541567, NASA NNG06GC89G; BDP by NASA grants NAS5-38099 (TRACE), NNM07AA01C (Hinode) and NNG06GG79G. SWM and BDP are jointly supported by NASA grants NNX08AL22G and NNX08AH45G. RJL is partially supported by NASA grant NNH08CC02C.

\section{References}

Attrill, G.D.R., van Driel-Gesztelyi, L., Démoulin, P., Zhukov, A.N., Steed, K., Harra, L.K., Mandrini, C.H., Linker, J.: 2008, The recovery of CME-related dimmings and the ICME's enduring magnetic connection to the Sun. Solar Phys. 252, 349. doi:10.1007/s11207-008-9255-z.

Bewsher, D., Harrison, R.A., Brown, D.S.: 2008, The relationship between EUV dimming and coronal mass ejections. I. Statistical study and probability model. Astron. Astrophys. 478, 897. doi:10.1051/0004-6361:20078615.

Brown, C.M., Feldman, U., Seely, J.F., Korendyke, C.M., Hara, H.: 2008, Wavelengths and intensities of spectral lines in the $171-211$ and 245-291 Å ranges from five solar regions recorded by the ExtremeUltraviolet Imaging Spectrometer (EIS) on Hinode. Astrophys. J. 176, 511. doi:10.1086/529378.

Cranmer, S.R., van Ballegooijen, A.A., Edgar, R.J.: 2007, Self-consistent coronal heating and solar wind acceleration from anisotropic magnetohydrodynamic turbulence. Astrophys. J. 171, 520. doi:10.1086/518001.

Culhane, J.L., Harra, L.K., James, A.M., Al-Janabi, K., Bradley, L.J., Chaudry, R.A., Rees, K., Tandy, J.A., Thomas, P., Whillock, M.C.R., Winter, B., Doschek, G.A., Korendyke, C.M., Brown, C.M., Myers, S., Mariska, J., Seely, J., Lang, J., Kent, B.J., Shaughnessy, B.M., Young, P.R., Simnett, G.M., Castelli, C.M., Mahmoud, S., Mapson-Menard, H., Probyn, B.J., Thomas, R.J., Davila, J., Dere, K., Windt, D., Shea, J., Hagood, R., Moye, R., Hara, H., Watanabe, T., Matsuzaki, K., Kosugi, T., Hansteen, V., Wikstol, Ø.: 2007, The EUV imaging spectrometer for Hinode. Solar Phys. 243, 19. doi:10.1007/s01007-007-0293-1.

De Pontieu, B., McIntosh, S.W., Carlsson, M., Hansteen, V.H., Tarbell, T.D., Schrijver, C.J., Title, A.M., Shine, R.A., Tsuneta, S., Katsukawa, Y., Ichimoto, K., Suematsu, Y., Shimizu, T., Nagata, S.: 2007, Chromospheric Alfvénic waves strong enough to power the solar wind. Science 318, 1574. doi:10.1126/science.1151747.

De Pontieu, B., McIntosh, S.W., Hansteen, V.H., Schrijver, C.J.: 2009, Observing the roots of solar coronal heating - in the chromosphere. Astrophys. J. Lett. 701, 1. doi:10.1088/0004-637X/701/1/L1.

Delaboudinière, J., Artzner, G.E., Brunaud, J., Gabriel, A.H., Hochedez, J.F., Millier, F., Song, X.Y., Au, B., Dere, K.P., Howard, R.A., Kreplin, R., Michels, D.J., Moses, J.D., Defise, J.M., Jamar, C., Rochus, P., Chauvineau, J.P., Marioge, J.P., Catura, R.C., Lemen, J.R., Shing, L., Stern, R.A., Gurman, J.B., Neupert, W.M., Maucherat, A., Clette, F., Cugnon, P., van Dessel, E.L.: 1995, EIT: extreme-ultraviolet imaging telescope for the SOHO mission. Solar Phys. 162, 291. doi:10.1007/BF00733432.

Freeland, S.L., Handy, B.N.: 1998, Data analysis with the SolarSoft system. Solar Phys. 182, 497.

Harra, L.K.: 2009, On-disk signatures of eruptive activity from the Hinode mission. Adv. Space Res. 44, 446. doi:10.1016/j.asr.2009.04.020.

Harra, L.K., Hara, H., Imada, S., Young, P.R., Williams, D.R., Sterling, A.C., Korendyke, C., Attrill, G.D.R.: 2007, Coronal dimming observed with Hinode: Outflows related to a coronal mass ejection. Publ. Astron. Soc. Japan 59, 801.

Harrison, R.A., Lyons, M.: 2000, A spectroscopic study of coronal dimming associated with a coronal mass ejection. Astron. Astrophys. 358, 1097.

Howard, R.A., Moses, J.D., Vourlidas, A., Newmark, J.S., Socker, D.G., Plunkett, S.P., Korendyke, C.M., Cook, J.W., Hurley, A., Davila, J.M., Thompson, W.T., St Cyr, O.C., Mentzell, E., Mehalick, K., Lemen, J.R., Wuelser, J.P., Duncan, D.W., Tarbell, T.D., Wolfson, C.J., Moore, A., Harrison, R.A., Waltham, N.R., Lang, J., Davis, C.J., Eyles, C.J., Mapson-Menard, H., Simnett, G.M., Halain, J.P., Defise, J.M., Mazy, E., Rochus, P., Mercier, R., Ravet, M.F., Delmotte, F., Auchere, F., Delaboudiniere, J.P., Bothmer, V., Deutsch, W., Wang, D., Rich, N., Cooper, S., Stephens, V., Maahs, G., Baugh, R., McMullin, D., Carter, T.: 2008, Sun Earth Connection Coronal and Heliospheric Investigation (SECCHI). Space Sci. Rev. 136, 67. doi:10.1007/s11214-008-9341-4.

Imada, S., Hara, H., Watanabe, T., Kamio, S., Asai, A., Matsuzaki, K., Harra, L.K., Mariska, J.T.: 2007, Discovery of a temperature-dependent upflow in the plage region during a gradual phase of the X-class flare. Publ. Astron. Soc. Japan 59, 793.

Jin, M., Ding, M.D., Chen, P.F., Fang, C., Imada, S.: 2009, Coronal mass ejection induced outflows observed with Hinode/EIS. Astrophys. J. 702, 27. doi:10.1088/0004-637X/702/1/27. 
Kahler, S.W., Hudson, H.S.: 2001, Origin and development of transient coronal holes. J. Geophys. Res. 106, 29239. doi:10.1029/2001JA000127.

Kosugi, T., Matsuzaki, K., Sakao, T., Shimizu, T., Sone, Y., Tachikawa, S., Hashimoto, T., Minesugi, K., Ohnishi, A., Yamada, T., Tsuneta, S., Hara, H., Ichimoto, K., Suematsu, Y., Shimojo, M., Watanabe, T., Shimada, S., Davis, J.M., Hill, L.D., Owens, J.K., Title, A.M., Culhane, J.L., Harra, L.K., Doschek, G.A., Golub, L.: 2007, The Hinode (Solar-B) mission: An overview. Solar Phys. 243, 3. doi:10.1007/s11207-007-9014-6.

Lites, B., Casini, R., Garcia, J., Socas-Navarro, H.: 2007, A suite of community tools for spectro-polarimetric analysis. Mem. Soc. Astron. Ital. 78, 148.

McIntosh, S.W.: 2009, The inconvenient truth about coronal dimmings. Astrophys. J. 693, 1306. doi:10.1088/0004-637X/693/2/1306.

McIntosh, S.W., De Pontieu, B.: 2009, Observing episodic coronal heating events rooted in chromospheric activity. Astrophys. J. 706, 80. doi:10.1088/0004-637X/706/1/L80.

McIntosh, S.W., Burkepile, J., Leamon, R.J.: 2009, More of the inconvenient truth about coronal dimmings. In: Proc. 2nd Hinode Science Meeting, in press. arXiv:0901.2817.

McIntosh, S.W., Davey, A.R., Hassler, D.M.: 2006, Simple magnetic flux balance as an indicator of Ne VIII Doppler velocity partitioning in an equatorial coronal hole. Astrophys. J. Lett. 644, 87. doi:10.1086/505488.

McIntosh, S.W., Leamon, R.J., Davey, A.R., Wills-Davey, M.J.: 2007, The posteruptive evolution of a coronal dimming. Astrophys. J. 660, 1653. doi:10.1086/512665.

McIntosh, S.W., Innes, D.E., De Pontieu, B., Leamon, R.J.: 2010, STEREO observations of quasiperiodically driven high velocity outflows in polar plumes. Astron. Astrophys. 510, L2. doi:10.1051/ 0004-6361/200913699.

Neugebauer, M., Goldstein, R., Goldstein, B.E.: 1997, Features observed in the trailing regions of interplanetary clouds from coronal mass ejections. J. Geophys. Res. 102, 19743. doi:10.1029/97JA01651.

Parker, E.N.: 1991, Heating solar coronal holes. Astrophys. J. 372, 719. doi:10.1086/170015.

Reinard, A.A., Biesecker, D.A.: 2009, The relationship between coronal dimming and coronal mass ejection properties. Astrophys. J. 705, 914. doi:10.1088/0004-637X/705/1/914.

Robbrecht, E., Berghmans, D.: 2004, Automated recognition of coronal mass ejections (CMEs) in near-realtime data. Astron. Astrophys. 425, 1097. doi:10.1051/0004-6361:20041302.

Scherrer, P.H., Bogart, R.S., Bush, R.I., Hoeksema, J.T., Kosovichev, A.G., Schou, J., Rosenberg, W., Springer, L., Tarbell, T.D., Title, A., Wolfson, C.J., Zayer, I., MDI Engineering Team: 1995, The solar oscillations investigation - Michelson Doppler imager. Solar Phys. 162, 129. doi:10.1007/BF00733429.

Skoug, R.M., Gosling, J.T., Steinberg, J.T., McComas, D.J., Smith, C.W., Ness, N.F., Hu, Q., Burlaga, L.F.: 2004, Extremely high speed solar wind: 29-30 October 2003. J. Geophys. Res. (Space Phys.) 109(A18), 9102. doi:10.1029/2004JA010494.

Skumanich, A., Lites, B.W.: 1987, Stokes profile analysis and vector magnetic fields. I - Inversion of photospheric lines. Astrophys. J. 322, 473. doi:10.1086/165743.

Suzuki, T.K., Inutsuka, S.: 2006, Solar winds driven by nonlinear low-frequency Alfvén waves from the photosphere: Parametric study for fast/slow winds and disappearance of solar winds. J. Geophys. Res. (Space Phys.) 111(A10), 6101. doi:10.1029/2005JA011502.

Verdini, A., Velli, M.: 2007, Alfvén waves and turbulence in the solar atmosphere and solar wind. Astrophys. J. 662, 669. doi:10.1086/510710. 